\title{
Prediction of thermodynamic properties: centerpiece approach—how do we avoid confusion and get reliable results?
}

\author{
Sergey P. Verevkin ${ }^{1,2}$ (1) $\cdot$ Irina V. Andreeva ${ }^{1} \cdot$ Kseniya V. Zherikova ${ }^{3} \cdot$ Aleksey A. Pimerzin $^{2}$
}

Received: 20 May 2021 / Accepted: 19 October 2021 / Published online: 11 December 2021

(C) The Author(s) 2021

\section{Abstract}

The absolute vapor pressures of three amino-alcohols were measured using the transpiration method. The consistent set of standard molar enthalpies of vaporization for eighteen amino-alcohols was evaluated using empirical and structure-property correlations. The averaged values of vaporization enthalpies were recommended as reliable benchmark properties for the heat management of $\mathrm{CO}_{2}$ capture technologies. Centerpiece approach based on the group-additivity principles was developed toward amino-alcohols.

\section{Graphic abstract}

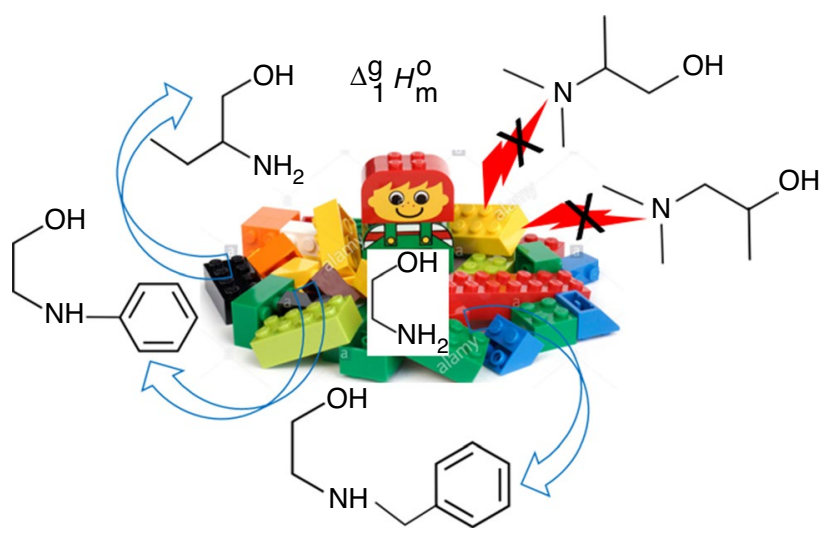

Keywords Amino-alcohols $\cdot$ Vapor pressure $\cdot$ Enthalpy of vaporization $\cdot$ Structure-property relationships

Sergey P. Verevkin

sergey.verevkin@uni-rostock.de

1 Department of Physical Chemistry and Faculty of Interdisciplinary Research, Competence Centre CALOR, University of Rostock, 18059 Rostock, Germany

2 Chemical Department, Samara State Technical University, Samara, Russia 443100

3 Nikolaev Institute of Inorganic Chemistry of Siberian Branch of Russian Academy of Sciences, Novosibirsk, Russia 630090

\section{Introduction}

The group-additivity (GA) approach offers an excellent way of assessing the thermodynamic properties of molecules whose properties have not been measured. One of the most popular GA approaches was developed by Sydney W. Benson [1]. A group is defined by Benson as "a polyvalent atom (ligancy $\geq 2$ ) in a molecule together with all of its ligands." The sum of the groups that constitute a molecule of interest provides a quick appraisal of a thermodynamic property. The GA works well for the gas-phase standard molar enthalpies of formation $\Delta_{\mathrm{f}} H_{\mathrm{m}}^{\mathrm{o}}(\mathrm{g}, 298.15 \mathrm{~K})$. The GA performance for the liquid-phase enthalpies of formation, $\Delta_{\mathrm{f}} H_{\mathrm{m}}^{\mathrm{o}}($ liq, $298.15 \mathrm{~K}$ ), is less successful because 
the intermolecular interactions between the molecules are randomly distributed among the groups. This fact aggravates the accuracy of the prediction considerably. In contrast, the standard molar enthalpies of vaporization, $\Delta_{\mathrm{f}} H_{\mathrm{m}}^{\mathrm{o}}$ $(298.15 \mathrm{~K})$, obey sufficiently well to the additivity rules. An important step in this direction was made by Ducros and co-workers [2] who showed that group additivity can be used to estimate the $\Delta_{\mathrm{f}} H_{\mathrm{m}}^{\mathrm{o}}(298.15 \mathrm{~K})$-values of organic and organometallic compounds with good precision. In his later work [3], Sydney W. Benson also expanded the GA method for calculating enthalpy of vaporization. We endorsed and followed Benson's approach and re-evaluated the group contributions using the updated $\Delta_{\mathrm{f}} H_{\mathrm{m}}^{\mathrm{o}}(\mathrm{g}, 298.15 \mathrm{~K}), \Delta_{\mathrm{f}} H_{\mathrm{m}}^{\mathrm{o}}$ (liq, $298.15 \mathrm{~K})$, and $\Delta_{\mathrm{f}} H_{\mathrm{m}}^{\mathrm{o}}(298.15 \mathrm{~K}$ ) data [4]. The crucial advantage of the Benson's method is that the energetics of a molecule of interest can be collected from scratch. However, many interactions between nearest and non-nearest neighbor groups, between substituents, and between fragments of the molecule are not taken into account by this procedure [5]. In the original Benson's scheme, such interactions are included as a list of individual "non-additive" contributions. However, the variety of possible structures of organic compounds is countless, so that the list of individual "non-additive" contributions can be endless.

In our most recent work [6, 7], we develop a "centerpiece" approach that is closely related to the conventional group-contribution methods $[3,4]$. In the latter methods, the molecule of interest is collected completely from welldefined group contributions. In contrast, the idea of this "centerpiece" approach is to select a "core" molecule that may possibly close to mimic the structure of the molecule of interest, but the selected "centerpiece" molecule has the well-established thermodynamic properties. Different substituents can be attached (or subtracted) to this "centerpiece" in different positions. The visualization of the "centerpiece" approach is given in Fig. 1.

For the prediction, e.g., the enthalpy of vaporization of 2-(phenyl-amino)-ethanol, a similarly shaped 2-(methylamino)-ethanol, can be seen as a well-suited "centerpiece." Indeed, the latter molecule already bears the energetic contribution due to the inherent intra-molecular hydrogen bond (intra-HB), which is the most significant feature of aminoethanols. Such a contribution is unique to the "centerpiece"
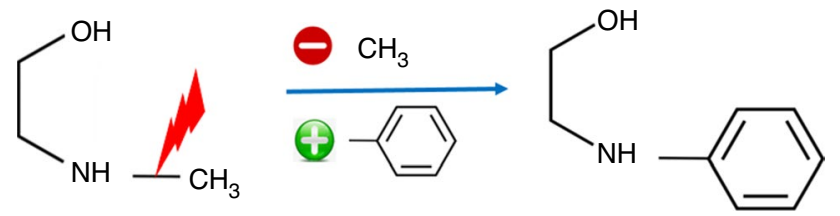

Fig. 1 Calculations of vaporization enthalpy, $\Delta_{1}^{\mathrm{g}} H_{\mathrm{m}}^{\mathrm{o}}(298.15 \mathrm{~K})$, of 2-(phenyl-amino)-ethanol from 2-(methyl-amino)-ethanol using the "centerpiece" approach molecule and cannot be captured by any other method. This special feature of the "centerpiece" approach increases the reliability of the property prediction for the similarly shaped molecules significantly. The experimental vaporization enthalpy of 2-(methyl-amino)-ethanol is well established in the literature $[8,9]$. Using this value as the "centerpiece," we can therefore exchange the methyl-group $\mathrm{CH}_{3}$ with the phenyl-group $\mathrm{C}_{6} \mathrm{H}_{5}$ and estimate the desired $\Delta_{1}^{\mathrm{g}} H_{\mathrm{m}}^{\mathrm{o}}(298.15 \mathrm{~K})$ value for of 2-(phenyl-amino)-ethanol. It should be noted that the conventional GA method to such predictions will not work because intra-HB is not parameterized at all. To validate the "centerpiece" approach to amino-alcohols, we examined the series of amino-alcohols shown in Fig. 2.

The focus of this work was on vapor pressure measurements and the standard molar vaporization enthalpies, $\Delta_{1}^{\mathrm{g}} H_{\mathrm{m}}^{\mathrm{o}}$ (298.15 K). We used different empirical and structure-property correlation methods for validation and evaluation of new and available experimental results.

We used the evaluated $\Delta_{1}^{\mathrm{g}} H_{\mathrm{m}}^{\mathrm{o}}(298.15 \mathrm{~K})$ values for aminoalcohols listed in Fig. 2 in order to show the success and limitations of the "centerpiece" approach.

\section{Experimental part}

\section{Materials}

All samples of amino-alcohols with purities of 0.96-0.99 mass fraction according to specification by manufacturer were of commercial origin (see Table S1). Samples were purified by repeated distillation at reduced pressures. Purities of samples were measured by a gas chromatograph equipped with FID and a HP-5 capillary column. No

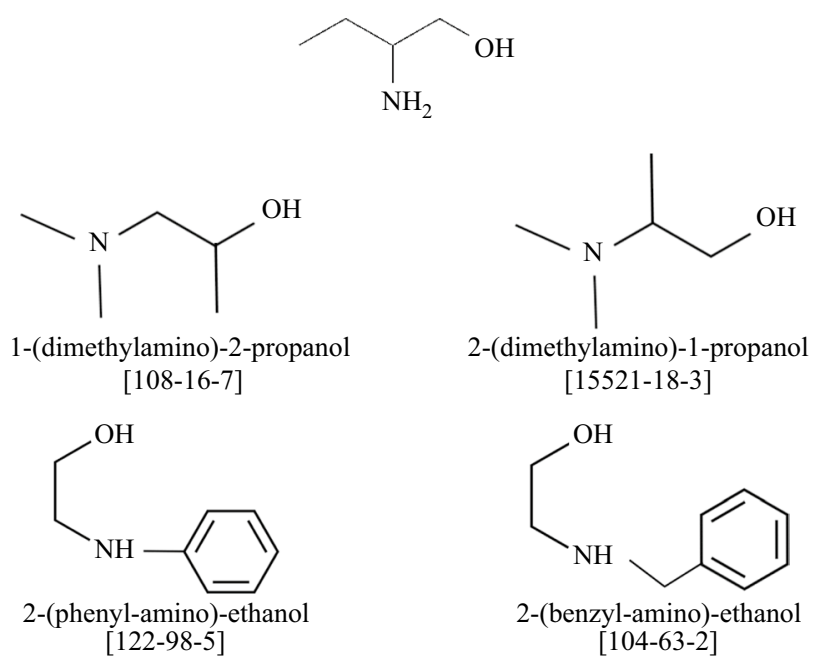

Fig. 2 Branched and phenyl-substituted amino-alcohols studied in this work 
impurities (greater than the mass fraction 0.0004) were found in the compounds studied by the transpiration.

\section{Experimental thermochemical methods}

Vapor pressures of 1-(dimethylamino)-2-propanol, 2-(phenyl-amino)-ethanol, and 2-(benzyl-amino)-ethanol at different temperatures were measured by using the transpiration method with a self-made setup [10,11]. Vapor pressures derived from the transpiration method are reliable within (1 to 3) $\%[10,11]$. The standard molar enthalpies of vaporization, $\Delta_{1}^{\mathrm{g}} H_{\mathrm{m}}^{\mathrm{o}}$, were derived from the temperature dependence of vapor pressure. The error in the enthalpies of vaporization is assumed to be within $\pm(0.3$ to 0.5$) \mathrm{kJ} \cdot \mathrm{mol}^{-1}[10,11]$. For more details on the experimental procedure, see the Supporting Information.

\section{Results and discussion}

\section{Absolute vapor pressures and thermodynamics of vaporization}

The experimental absolute vapor pressures, $p_{i}$, and temperature dependences measured with the help of the transpiration method for amino-alcohols were fitted with the following equation [10]:

$R \times \ln \left(p_{\mathrm{i}} / p_{\text {ref }}\right)=a+\frac{b}{T}+\Delta_{1}^{\mathrm{g}} C_{\mathrm{p}, \mathrm{m}}^{\mathrm{o}} \times \ln \left(\frac{T}{T_{0}}\right)$

where $a$ and $b$ are adjustable parameters, the reference pressure $p_{\text {ref }}=1 \mathrm{~Pa}$, and $R=8.31446 \mathrm{~J} \cdot \mathrm{K}^{-1} \cdot \mathrm{mol}^{-1}$. The $\Delta_{1}^{\mathrm{g}} C_{\mathrm{p}, \mathrm{m}}^{\mathrm{o}}$ is the difference of the molar heat capacities of the gas and the liquid phases, respectively (see Table S2). The arbitrary temperature $T_{0}$ applied in Eq. (1) was chosen to be $T_{0}=298.15 \mathrm{~K}$.

The primary experimental results on vapor pressures for the DL-2-amino-1-butanol, 1-(dimethylamino)-2-propanol, 2-(phenyl-amino)-ethanol, and 2-(benzyl-amino)-ethanol from the transpiration method are summarized in Table 1.

The vapor pressures for DL-2-amino-1-butanol and 1-(dimethylamino)-2-propanol were measured for the first time. The vapor pressures at different temperatures for 2-(phenyl-amino)-ethanol are reported by Stull [47]. These data correspond to the significantly higher temperature range, and a comparison is not possible. The vapor pressure temperature dependence for 2-(benzyl-amino)-ethanol was measured by Razzouk et al. [15] using the static method. The comparison of the available data is given in Fig. S1. Our transpiration results are significantly lower compared to those of the static technique. It has turned out that the purity of the sample used by Razzouk et al. [15] was only $97.7 \%$ according to gas chromatography as determined after vapor pressure measurements. The information of the remaining $2.3 \%$ impurities is absent in the paper. In our experience with the static method, however, these impurities should be the decomposition product with higher volatility and could significantly increase the vapor pressure. Nevertheless, this apparent disagreement led us to look for additional data that would help resolve the observed contradictions. As a matter of fact, the SciFinder [16] compiles experimental boiling temperatures at various pressures. The accuracy of this data is questionable as it comes from the distillation of a compound after its synthesis and not from special physicochemical studies. However, the numerous data on boiling temperatures at standard pressure, as well as at reduced pressures, provide at least a reliable level of the experimental vapor pressures and a reliable trend of the dependence of the vapor pressure temperature. As can be seen in Fig. S1, the boiling points taken from SciFinder agree fair with our transpiration results, but not with those from the static method. After we have resolved the contradictions observed in this way for 2-(benzyl-amino)-ethanol, we have systematically (see Table S3) collected the data available in SciFinder for aminoalcohols (see Fig. 2). These data were used to derive vaporization enthalpies of amino-alcohols (see Table 2). The "empirical" results derived in this way are designated in Table 2 as $S F$ values.

Vapor pressure temperature dependences measured in this work (and those taken from the literature) were used to derive vaporization enthalpies according to the following equation:

$\Delta_{1}^{\mathrm{g}} H_{\mathrm{m}}^{\mathrm{o}}(T)=-b+\Delta_{1}^{\mathrm{g}} C_{\mathrm{p}, \mathrm{m}}^{\mathrm{o}} \times T$

The entropies of vaporization were also obtained from vapor pressures temperature dependences according to Eq. (3):

$\Delta_{1}^{\mathrm{g}} S_{\mathrm{m}}^{\mathrm{o}}(T)=\Delta_{1}^{\mathrm{g}} H_{\mathrm{m}}^{\mathrm{o}} / T+R \times \ln \left(p_{\mathrm{i}} / p^{\mathrm{o}}\right)$

with $p^{\mathrm{o}}=0.1 \mathrm{MPa}$. Values of $\Delta_{1}^{\mathrm{g}} H_{\mathrm{m}}^{\mathrm{o}}(T)$ and $\Delta_{1}^{\mathrm{g}} S_{\mathrm{m}}^{\mathrm{o}}(T)$ are collected in Table 1. The procedure for calculating the combined uncertainties of enthalpy of vaporization has been described elsewhere $[12,13]$. The uncertainties include uncertainties in vapor pressure, uncertainties due to the experimental conditions of transpiration, and uncertainties in adjustment to $T=298.15 \mathrm{~K}$. The compilation of the $\Delta_{1}^{\mathrm{g}} H_{\mathrm{m}}^{\mathrm{o}}$ (298.15 K) values of amino-alcohols, calculated according to Eq. (2), is given in Table 2.

\section{Correlation of enthalpies of vaporization of amino-alcohols with their boiling temperatures}

The relation of vaporization enthalpy to boiling point is a well-established phenomenon, and Trouton's rule is the 
Table 1 Vapor pressures $p$, of amino-alcohols measured by transpiration, standard $\left(p^{\mathrm{o}}=0.1 \mathrm{MPa}\right)$ molar enthalpies of vaporization and standard $\left(p^{\mathrm{o}}=0.1 \mathrm{MPa}\right)$ molar entropies of vaporization

\begin{tabular}{|c|c|c|c|c|c|c|c|c|}
\hline$T / \mathrm{K}^{\mathrm{a}}$ & $m / \mathrm{mg}^{\mathrm{b}}$ & $V\left(\mathrm{~N}_{2}\right)^{\mathrm{c}} / \mathrm{dm}^{3}$ & $T_{\mathrm{a}} / \mathrm{K}^{\mathrm{d}}$ & Flow $/ \mathrm{dm}^{3} \mathrm{~h}^{-1}$ & $p / \mathrm{Pa}$ & $u(p) / \mathrm{Pa}^{\mathrm{f}}$ & $\begin{array}{l}\Delta_{\mathrm{l}, \mathrm{cr}}^{\mathrm{g}} H_{\mathrm{m}}^{\mathrm{o}} \\
(T) \mathrm{kJ} \\
\mathrm{mol}^{-1}\end{array}$ & $\begin{array}{l}\Delta_{\mathrm{l}, \mathrm{c}}^{\mathrm{g}} S_{\mathrm{m}}^{\mathrm{o}}(T) \mathrm{J} \\
\mathrm{K}^{-\mathrm{I}} \mathrm{mol}^{-1}\end{array}$ \\
\hline
\end{tabular}

DL-2-amino-1-butanol [13054-87-0]: $\Delta_{1}^{\mathrm{g}} H_{\mathrm{m}}^{\mathrm{o}}$

$(298.15 \mathrm{~K})=(65.5 \pm 0.5) \mathrm{kJ} \cdot \mathrm{mol}^{-1}$

$\ln \left(p / p_{\text {ref }}\right)=\frac{317.8}{R}-\frac{86891.6}{R T}-\frac{71.8}{R} \ln \frac{T}{298.15} ; p_{\text {ref }}=1 \mathrm{~Pa}$ 303.2

308.3

$\begin{array}{llll}1.45 & 1.073 & 295.8 & 1.31\end{array}$

$\begin{array}{llll}1.79 & 0.898 & 295.8 & 1.31\end{array}$

313.4

318.2

323.4

328.4

333.5

328.4

333.5

1-(Dimethyl-amino)-2-propanol [108-16-7]: $\Delta_{1}^{\mathrm{g}} H_{\mathrm{m}}^{\mathrm{o}}$

$\begin{array}{llll}2.20 & 0.712 & 295.8 & 1.31\end{array}$

$\begin{array}{llll}2.71 & 0.613 & 295.8 & 1.31\end{array}$

$\begin{array}{llll}2.33 & 0.350 & 295.8 & 1.31\end{array}$

$\begin{array}{llll}3.25 & 0.339 & 295.8 & 1.31\end{array}$

$\begin{array}{llll}3.66 & 0.274 & 295.8 & 1.31\end{array}$

$\begin{array}{llll}3.25 & 0.339 & 295.8 & 1.31\end{array}$

$\begin{array}{llll}3.66 & 0.274 & 295.8 & 1.31\end{array}$

$\begin{array}{clll}37.34 & 0.96 & 65.1 & 149.2 \\ 55.13 & 1.40 & 64.8 & 147.7 \\ 85.45 & 2.16 & 64.4 & 146.8 \\ 121.7 & 3.1 & 64.0 & 145.5 \\ 183.5 & 4.6 & 63.7 & 144.5 \\ 263.4 & 6.6 & 63.3 & 143.4 \\ 367.6 & 9.2 & 62.9 & 142.2 \\ 263.4 & 6.6 & 63.3 & 143.4 \\ 367.6 & 9.2 & 62.9 & 142.2\end{array}$

$(298.15 \mathrm{~K})=(45.7 \pm 0.3) \mathrm{kJ}^{\circ} \mathrm{mol}^{-1}$

$\ln \left(p / p_{\text {ref }}\right)=\frac{287.4}{R}-\frac{67352.5}{R T}-\frac{72.7}{R} \ln \frac{T}{298.15} ; p_{\text {ref }}=1 \mathrm{~Pa}$

276.2

276.3

276.3

277.7

277.7

279.2

279.3

280.7

281.9

282.3

282.3

284.1

284.7

285.2

285.2

286.3

287.2

287.7

288.2

289.1

289.1

290.1

291.2

291.2

294.2

297.2

300.2

303.2

306.2

308.2

2-(Phenyl-amino)-ethanol [122-98-5]: $\Delta_{1}^{\mathrm{g}} H_{\mathrm{m}}^{\mathrm{o}}$

$(298.15 \mathrm{~K})=(82.3 \pm 0.4) \mathrm{kJ}^{2} \mathrm{~mol}^{-1}$

$\begin{array}{rlll}5.73 & 0.396 & 295.7 & 0.91 \\ 6.28 & 0.432 & 295.7 & 1.00 \\ 6.23 & 0.422 & 295.7 & 0.97 \\ 6.28 & 0.389 & 295.7 & 0.93 \\ 5.43 & 0.335 & 295.7 & 0.91 \\ 5.33 & 0.292 & 295.7 & 0.97 \\ 5.53 & 0.305 & 295.7 & 0.91 \\ 5.58 & 0.273 & 295.7 & 0.91 \\ 6.93 & 0.305 & 295.7 & 0.91 \\ 6.18 & 0.268 & 295.7 & 0.97 \\ 6.03 & 0.267 & 295.7 & 0.91 \\ 6.68 & 0.251 & 295.7 & 0.91 \\ 7.30 & 0.267 & 295.7 & 0.92 \\ 5.23 & 0.187 & 295.7 & 0.93 \\ 6.58 & 0.236 & 295.7 & 0.91 \\ 10.43 & 0.340 & 295.7 & 1.00 \\ 6.18 & 0.190 & 295.7 & 0.91 \\ 6.63 & 0.198 & 295.7 & 0.92 \\ 6.03 & 0.175 & 295.7 & 0.91 \\ 9.18 & 0.249 & 295.7 & 1.00 \\ 9.43 & 0.257 & 295.7 & 1.00 \\ 6.38 & 0.160 & 295.7 & 0.91 \\ 5.93 & 0.140 & 295.7 & 0.93 \\ 6.33 & 0.152 & 295.7 & 0.91 \\ 5.93 & 0.114 & 295.7 & 0.91 \\ 6.89 & 0.107 & 295.7 & 0.92 \\ 12.69 & 0.163 & 295.7 & 0.93 \\ 14.44 & 0.152 & 295.7 & 0.91 \\ 12.06 & 0.107 & 295.7 & 0.92 \\ 13.61 & 0.107 & 295.7 & 0.92 \\ & & & \\ 6 & & \\ 6.91\end{array}$

$367.1 \quad 9.2$

47.3

124.6

$369.1 \quad 9.3$

47.3

124.5

$374.1 \quad 9.4$

$47.3 \quad 124.6$

$\begin{array}{llll}407.0 & 10.2 & 47.2 & 124.1\end{array}$

$\begin{array}{llll}408.0 & 10.2 & 47.2 & 124.1\end{array}$

$\begin{array}{llll}456.2 & 11.4 & 47.1 & 123.8\end{array}$

$\begin{array}{llll}454.1 & 11.4 & 47.1 & 123.6\end{array}$

$\begin{array}{llll}508.1 & 12.7 & 46.9 & 123.4\end{array}$

$\begin{array}{llll}562.7 & 14.1 & 46.9 & 123.2\end{array}$

$\begin{array}{llll}570.3 & 14.3 & 46.8 & 123.0\end{array}$

$\begin{array}{llll}559.6 & 14.0 & 46.8 & 122.8\end{array}$

$\begin{array}{llll}652.9 & 16.3 & 46.7 & 122.6\end{array}$

$\begin{array}{llll}671.7 & 16.8 & 46.7 & 122.3\end{array}$

$\begin{array}{llll}686.6 & 17.2 & 46.6 & 122.1\end{array}$

$\begin{array}{llll}683.2 & 17.1 & 46.6 & 122.0\end{array}$

$\begin{array}{llll}749.1 & 18.8 & 46.5 & 121.9\end{array}$

$\begin{array}{llll}790.9 & 19.8 & 46.5 & 121.6\end{array}$

$\begin{array}{llll}814.6 & 20.4 & 46.4 & 121.5\end{array}$

$\begin{array}{llll}836.9 & 20.9 & 46.4 & 121.3\end{array}$

$\begin{array}{llll}894.9 & 22.4 & 46.3 & 121.1\end{array}$

$\begin{array}{llll}889.8 & 22.3 & 46.3 & 121.1\end{array}$

$\begin{array}{llll}965.0 & 24.1 & 46.3 & 120.9\end{array}$

$\begin{array}{llll}1022.9 & 25.6 & 46.2 & 120.5\end{array}$

$\begin{array}{llll}1008.1 & 25.2 & 46.2 & 120.4\end{array}$

$\begin{array}{llll}1250.2 & 31.3 & 46.0 & 119.8\end{array}$

$\begin{array}{llll}1538.7 & 38.5 & 45.7 & 119.3\end{array}$

$\begin{array}{llll}1842.2 & 46.1 & 45.5 & 118.5\end{array}$

$\begin{array}{llll}2233.4 & 55.9 & 45.3 & 117.9\end{array}$

$\begin{array}{llll}2645.6 & 66.2 & 45.1 & 117.1\end{array}$

$\begin{array}{llll}2972.9 & 74.3 & 44.9 & 116.6\end{array}$ 
Table 1 (continued)

\begin{tabular}{|c|c|c|c|c|c|c|c|c|}
\hline$T / \mathrm{K}^{\mathrm{a}}$ & $m / \mathrm{mg}^{\mathrm{b}}$ & $V\left(\mathrm{~N}_{2}\right)^{\mathrm{c}} / \mathrm{dm}^{3}$ & $T_{\mathrm{a}} / \mathrm{K}^{\mathrm{d}}$ & Flow $/ \mathrm{dm}^{3} \mathrm{~h}^{-1}$ & $p / \mathrm{Pa}^{\mathrm{e}}$ & $u(p) / \mathrm{Pa}^{\mathrm{f}}$ & $\begin{array}{l}\Delta_{\mathrm{l}, \mathrm{cr}}^{\mathrm{g}} H_{\mathrm{m}}^{\mathrm{o}} \\
(T) \mathrm{kJ} \\
\mathrm{mol}^{-1}\end{array}$ & $\begin{array}{l}\Delta_{\mathrm{l,cr}}^{\mathrm{g}} S_{\mathrm{m}}^{\mathrm{o}}(T) \mathrm{J} \\
\mathrm{K}^{, \mathrm{c}} \mathrm{mol}^{-1}\end{array}$ \\
\hline \multicolumn{9}{|c|}{$\ln \left(p / p_{\text {ref }}\right)=\frac{342.6}{R}-\frac{107623.8}{R T}-\frac{85.0}{R} \ln \frac{T}{298.15} ; p_{\text {ref }}=1 \mathrm{~Pa}$} \\
\hline 308.1 & 1.34 & 73.79 & 293.8 & 4.31 & 0.32 & 0.01 & 81.4 & 159.2 \\
\hline 311.1 & 1.88 & 78.96 & 293.8 & 4.31 & 0.42 & 0.02 & 81.2 & 158.2 \\
\hline 314.1 & 1.36 & 41.85 & 293.8 & 4.31 & 0.58 & 0.02 & 80.9 & 157.5 \\
\hline 317.2 & 1.38 & 30.36 & 293.8 & 4.31 & 0.81 & 0.03 & 80.7 & 156.9 \\
\hline 320.1 & 1.43 & 25.05 & 293.8 & 4.31 & 1.02 & 0.03 & 80.4 & 155.7 \\
\hline 321.1 & 1.50 & 23.11 & 293.8 & 4.31 & 1.16 & 0.03 & 80.3 & 155.7 \\
\hline 324.1 & 2.05 & 24.62 & 293.8 & 4.31 & 1.48 & 0.04 & 80.1 & 154.7 \\
\hline 327.2 & 1.91 & 17.37 & 293.8 & 4.31 & 1.96 & 0.05 & 79.8 & 153.8 \\
\hline 330.2 & 1.91 & 12.92 & 293.8 & 4.31 & 2.63 & 0.07 & 79.6 & 153.3 \\
\hline 333.1 & 1.50 & 7.968 & 293.8 & 4.31 & 3.35 & 0.09 & 79.3 & 152.5 \\
\hline 336.2 & 1.51 & 6.173 & 293.8 & 4.31 & 4.37 & 0.11 & 79.1 & 151.7 \\
\hline 338.2 & 0.82 & 2.871 & 293.8 & 4.31 & 5.07 & 0.15 & 78.9 & 151.0 \\
\hline 338.2 & 1.84 & 6.481 & 293.8 & 4.52 & 5.06 & 0.15 & 78.9 & 151.0 \\
\hline 341.2 & 1.77 & 4.747 & 293.8 & 4.52 & 6.66 & 0.19 & 78.6 & 150.5 \\
\hline 344.4 & 2.29 & 4.810 & 293.8 & 4.31 & 8.49 & 0.24 & 78.4 & 149.6 \\
\hline 348.5 & 1.41 & 2.082 & 293.8 & 4.31 & 12.02 & 0.33 & 78.0 & 148.8 \\
\hline \multicolumn{9}{|c|}{$\begin{array}{l}\text { 2-(Benzyl-amino)-ethanol: } \Delta_{1}^{\mathrm{g}} H_{\mathrm{m}}^{\mathrm{o}} \\
\qquad(298.15 \mathrm{~K})=(84.5 \pm 0.4) \mathrm{kJ} \cdot \mathrm{mol}^{-1}\end{array}$} \\
\hline \multicolumn{9}{|c|}{$\ln \left(p / p_{\text {ref }}\right)=\frac{360.7}{R}-\frac{112301.0}{R T}-\frac{93.3}{R} \ln \frac{T}{298.15} ; p_{\text {ref }}=1 \mathrm{~Pa}$} \\
\hline 302.2 & 0.98 & 68.47 & 294.0 & 4.47 & 0.23 & 0.01 & 84.1 & 170.5 \\
\hline 305.1 & 1.15 & 59.23 & 294.0 & 4.36 & 0.31 & 0.01 & 83.8 & 169.5 \\
\hline 308.1 & 1.64 & 58.81 & 294.0 & 4.50 & 0.45 & 0.02 & 83.6 & 168.9 \\
\hline 311.1 & 1.04 & 28.56 & 294.0 & 4.36 & 0.59 & 0.02 & 83.3 & 167.6 \\
\hline 314.0 & 1.09 & 22.31 & 294.0 & 4.36 & 0.79 & 0.02 & 83.0 & 166.7 \\
\hline 317.1 & 1.93 & 29.33 & 294.0 & 4.50 & 1.06 & 0.03 & 82.7 & 165.7 \\
\hline 319.0 & 1.06 & 12.90 & 294.0 & 4.50 & 1.33 & 0.04 & 82.5 & 165.4 \\
\hline 321.0 & 0.77 & 7.682 & 294.0 & 4.47 & 1.63 & 0.05 & 82.4 & 164.9 \\
\hline 323.1 & 0.76 & 6.323 & 294.0 & 4.36 & 1.95 & 0.05 & 82.2 & 164.2 \\
\hline 326.1 & 1.15 & 7.195 & 294.0 & 4.36 & 2.58 & 0.07 & 81.9 & 163.3 \\
\hline 329.1 & 1.37 & 6.541 & 294.0 & 4.36 & 3.39 & 0.09 & 81.6 & 162.4 \\
\hline 331.1 & 0.94 & 3.655 & 294.0 & 4.47 & 4.16 & 0.11 & 81.4 & 162.0 \\
\hline 332.1 & 1.30 & 4.724 & 294.0 & 4.36 & 4.46 & 0.12 & 81.3 & 161.6 \\
\hline 335.1 & 1.05 & 2.907 & 294.0 & 4.36 & 5.83 & 0.17 & 81.0 & 160.8 \\
\hline 338.2 & 0.97 & 2.035 & 294.0 & 4.36 & 7.71 & 0.22 & 80.8 & 160.1 \\
\hline 341.2 & 0.91 & 1.526 & 294.0 & 4.36 & 9.65 & 0.27 & 80.5 & 159.0 \\
\hline 344.4 & 1.08 & 1.381 & 294.0 & 4.36 & 12.62 & 0.34 & 80.2 & 158.2 \\
\hline
\end{tabular}

${ }^{\text {a }}$ Saturation temperature, $(u(T)=0.1 \mathrm{~K})$

${ }^{\mathrm{b}}$ Mass of sample transferred and condensed at $T=243 \mathrm{~K}$

${ }^{\mathrm{c}}$ Volume of carrier gas $\left(u(V)=0.005 \mathrm{dm}^{3}\right)$ used to transfer $m(u(m)=0.0001 \mathrm{~g})$ of the sample

${ }^{\mathrm{d}} T_{\mathrm{a}}$ is the temperature of the soap bubble meter used for the measurement of the gas flow

${ }^{\text {e}}$ Vapor pressure at temperature $T$, calculated from the $m$ and the residual vapor pressure at the condensation temperature calculated by an iteration procedure

${ }^{\mathrm{f}}$ Uncertainties of absolute vapor pressures: $u\left(p_{i} / \mathrm{Pa}\right)=0.025+0.025\left(p_{i} / \mathrm{Pa}\right)$ for pressures from 5 to $3000 \mathrm{~Pa}$ and $u\left(p_{i} / \mathrm{Pa}\right)=0.005+0.025\left(p_{i} / \mathrm{Pa}\right)$ for pressures below $5 \mathrm{~Pa}$. Uncertainties of enthalpies of vaporization (standard uncertainties, 0.68 level of confidence, $\mathrm{k}=1$ ) are calculated as described elsewhere $[12,13]$ 
Table 2 The standard molar vaporization enthalpies, $\Delta_{1}^{\mathrm{g}} H_{\mathrm{m}}^{\mathrm{o}}$, of substituted amino-alcohols

\begin{tabular}{|c|c|c|c|c|}
\hline Compound & $\mathrm{M}^{\mathrm{a}}$ & $T$ - range & $\Delta_{1}^{\mathrm{g}} H_{\mathrm{m}}^{\mathrm{o}}\left(T_{\mathrm{av}}\right)$ & $\Delta_{\mathrm{l}}^{\mathrm{g}} H_{\mathrm{m}}^{\mathrm{o}}(298.15 \mathrm{~K})^{\mathrm{b}}$ \\
\hline & & $\mathrm{K}$ & $\mathrm{kJ} \cdot \mathrm{mol}^{-1}$ & $\mathrm{~kJ} \cdot \mathrm{mol}^{-1}$ \\
\hline 2-(Methyl-amino)-ethanol [109-83-1] & & & & $57.7 \pm 0.2[9]$ \\
\hline 2-(Ethyl-amino)-ethanol [110-73-6] & & & & $60.8 \pm 0.2[20]$ \\
\hline DL-2-amino-1-butanol & $\mathrm{T}$ & $303.2-333.5$ & $64.0 \pm 0.4$ & $65.5 \pm 0.5$ \\
\hline 1-(dimethylamino)-2-propanol & SF & $333-400$ & $42.3 \pm 0.6$ & $47.3 \pm 0.7$ \\
\hline \multirow[t]{2}{*}{ [108-16-7] } & $\mathrm{Tb}$ & 400 & & $44.9 \pm 1.5$ \\
\hline & $\mathrm{T}$ & $276.2-308.2$ & $46.3 \pm 0.2$ & $\begin{array}{l}45.7 \pm 0.3 \\
45.6 \pm 0.2^{c}\end{array}$ \\
\hline 2-(Dimethylamino)-1-propanol & SF & $341-423$ & $45.8 \pm 0.5$ & $51.6 \pm 0.7$ \\
\hline [15521-18-3] & $\mathrm{Tb}$ & 420.5 & & $\begin{array}{l}50.3 \pm 1.5 \\
\mathbf{5 1 . 4} \pm \mathbf{0 . 6}^{\mathrm{c}}\end{array}$ \\
\hline 2-(Phenyl-amino)-ethanol & $\mathrm{n} / \mathrm{a}$ & $377.2-552.8$ & $65.5 \pm 1.5$ & $78.8 \pm 1.7[14]$ \\
\hline \multirow[t]{5}{*}{ [122-98-5] } & SF & $383-560$ & $68.6 \pm 1.4$ & $83.6 \pm 1.6$ \\
\hline & $\mathrm{Tb}$ & 553 & & $85.0 \pm 1.5$ \\
\hline & Add & & & $83.3 \pm 1.0$ \\
\hline & $\mathrm{Jx}$ & & & $83.4 \pm 1.0$ \\
\hline & $\mathrm{T}$ & $308.1-348.5$ & $79.8 \pm 0.3$ & $\begin{array}{l}82.3 \pm 0.4 \\
82.6 \pm 0.3^{c}\end{array}$ \\
\hline 2-(Benzyl-amino)-ethanol & $S$ & $292.8-362.9$ & $71.7 \pm 0.6$ & $(74.5 \pm 0.7)[15]$ \\
\hline \multirow[t]{6}{*}{$104-63-2$} & SF & $379-562$ & $78.5 \pm 3.6$ & $92.4 \pm 3.7$ \\
\hline & $\mathrm{Tb}$ & $(562)$ & & $87.0 \pm 1.5$ \\
\hline & Add & & & $86.2 \pm 1.0$ \\
\hline & $\mathrm{J} \mathrm{X}$ & & & $83.5 \pm 1.0$ \\
\hline & $\mathrm{T}$ & $302.2-344.4$ & $82.2 \pm 0.3$ & $84.5 \pm 0.4$ \\
\hline & & & & $84.7 \pm 0.4^{c}$ \\
\hline
\end{tabular}

${ }^{a}$ Techniques: $\mathrm{T}=$ transpiration method; $\mathrm{S}=$ static method; $\mathrm{n} / \mathrm{a}=$ method is not available; $\mathrm{SF}-$ from experimental boiling temperatures reported at different pressures compiled by the SciFinder [16] (see text); $J_{x}-$ results obtained from Kovats indices (see text); Add = calculated according to the "centerpiece" approach

${ }^{b}$ Uncertainties are the standard uncertainty. They include uncertainties from the vapor pressure fitting equation and uncertainties from adjustment to the reference temperature $T=298.15 \mathrm{~K}[12,13]$

${ }^{\mathrm{c}}$ Weighted mean value with the standard uncertainty. The recommended thermochemical calculations values are highlighted in bold. Values in brackets were not included by the averaging best evidence of this. According to our experience, the aminoalcohols are thermally stable compounds, which boil between 400 and $560 \mathrm{~K}$ [17-19], depending on their size and structure. In our previous paper [20], we have established a linear correlations of $\Delta_{1}^{\mathrm{g}} H_{\mathrm{m}}^{\mathrm{o}}(298.15 \mathrm{~K})$-values with $T_{\mathrm{b}}$ values that were found for the set containing primary and secondary amino-alcohols $\left(\mathrm{kJ} \cdot \mathrm{mol}^{-1}\right)$ :

$\Delta_{1}^{\mathrm{g}} H_{\mathrm{m}}^{\mathrm{o}}(298.15 K)=-35.3+0.2193 \times \mathrm{T}_{\mathrm{b}} \quad$ with $\quad\left(R_{2}=0.966\right)$

The results calculated from this correlation were in agreement with those derived from other methods within of $\pm 1.5 \mathrm{~kJ} \cdot \mathrm{mol}^{-1}$. We used Eq. (4) to calculate vaporization enthalpies of 1-(dimethylamino)-2-propanol, 2-(dimethylamino)-1-propanol, 2-(phenyl-amino)-ethanol, and 2-(benzyl-amino)-ethanol, which are given in Table 2, and designated as the $T_{\mathrm{b}}$-values.

\section{Kovats retention indices for validation of experimental vaporization enthalpies}

In homologous series (e.g., alcohols, alkylbenzenes, alkanes, etc.), the vaporization enthalpies $\Delta_{1}^{\mathrm{g}} H_{\mathrm{m}}^{\mathrm{o}}(298.15 \mathrm{~K})$ show a linear correlation with Kovats indices [21, 22]. The high-quality linear correlation was derived when the $\Delta_{1}^{\mathrm{g}} H_{\mathrm{m}}^{\mathrm{o}}(298.15 \mathrm{~K})$-values are correlated with $J_{\mathrm{x}}$-values for the structurally parent set of amino-alcohols collected in Table 3: 
Table 3 Correlation of Kovats indices $\left(J_{\mathrm{x}}\right)$ and vaporization enthalpies, $\Delta_{1}^{\mathrm{g}} H_{\mathrm{m}}^{\mathrm{o}}(298.15 \mathrm{~K})$, of amino-alcohols

\begin{tabular}{llllll}
\hline & & $J_{\mathrm{x}}{ }^{\mathrm{a}}$ & $\Delta_{1}^{\mathrm{g}} H_{\mathrm{m}}^{\mathrm{o}}(298 \mathrm{~K})_{\exp }{ }^{\mathrm{b}}$ & $\Delta_{1}^{\mathrm{g}} H_{\mathrm{m}}^{\mathrm{o}}(298 \mathrm{~K})_{\mathrm{calc}^{\mathrm{c}}}$ & $\Delta^{\mathrm{d}}$ \\
\hline CAS & Compound & & $\mathrm{kJ} \mathrm{mol}^{-1}$ & $\mathrm{~kJ} \mathrm{~mol}^{-1}$ & $\mathrm{~kJ} \mathrm{~mol}^{-1}$ \\
$109-83-1$ & 2-(methyl-amino)-ethanol & 700 & $57.7 \pm 0.2[9]$ & 57.5 & 0.2 \\
$110-73-6$ & 2-(ethyl-amino)-ethanol & 786 & $60.8 \pm 0.2[20]$ & 61.0 & -0.2 \\
$122-98-5$ & 2-(phenyl-amino)-ethanol & 1347 & $82.3 \pm 0.4^{\mathrm{e}}$ & 83.3 & -1.1 \\
$104-63-2$ & 2-(benzyl-amino)-ethanol & 1349 & $84.5 \pm 0.4^{\mathrm{e}}$ & 83.4 & -1.1 \\
\hline
\end{tabular}

${ }^{a}$ Kovats indices, $J \mathrm{x}$, on the standard non-polar column OV-1[23]

${ }^{\mathrm{b}}$ Experimental data from Table 2

${ }^{c}$ Calculated using Eq. (5) with the assessed standard uncertainty of $\pm 1.0 \mathrm{~kJ} \cdot \mathrm{mol}^{-1}$

${ }^{\mathrm{d}}$ Difference between columns 4 and 5 in this table

${ }^{e}$ Experimental data measured by using the transpiration method (see Table 2)
$\Delta_{\mathrm{l}}^{\mathrm{g}} H_{\mathrm{m}}^{\mathrm{o}}(298.15 \mathrm{~K}) /\left(\mathrm{kJ} . \mathrm{mol}^{-1}\right)=29.6+0.0399 \times J_{\mathrm{x}}$ with $(R 2=0.996)$

The "theoretical" results derived from this correlation are given in Table 2 and designated as $J_{\mathrm{x}}$. The vaporization enthalpies derived from the correlations with Kovats indices (see Table 3, column 5) are in a good agreement with those obtained by the transpiration method (see Table 2).

Such good agreement can be seen as an additional validation of the experimental data measured in this work by using the transpiration method (see Table 2). It can be seen from Table 3 that differences between experimental and calculated according to Eq. (4) vaporization enthalpies are at the level of $1 \mathrm{~kJ} \cdot \mathrm{mol}^{-1}$ in the worst cases. Hence, the uncertainties of enthalpies of vaporization which are estimated from the correlation the $\Delta_{1}^{\mathrm{g}} H_{\mathrm{m}}^{\mathrm{o}}(298.15 \mathrm{~K})-J_{\mathrm{x}}$ are evaluated with $\pm 1.0 \mathrm{~kJ} \cdot \mathrm{mol}^{-1}$.

\section{Evaluation of available vaporization enthalpies}

As can be seen from Table 2, the vaporization enthalpies, $\Delta_{1}^{\mathrm{g}} H_{\mathrm{m}}^{\mathrm{o}}(298.15 \mathrm{~K})$, derived from vapor pressures measured by the conventional methods, as well as those derived from the SciFinder [16] data are in good agreement for amino-alcohols compiled in this table. Such a good agreement has reinforced usefulness of the experimental boiling temperatures reported at different pressures compiled by the SciFinder for evaluation of the scarce thermodynamic data.

An additional validation of the $\Delta_{1}^{\mathrm{g}} H_{\mathrm{m}}^{\mathrm{o}}(298.15 \mathrm{~K})$ values collected in Table 2 was conducted with the correlation $\Delta_{1}^{\mathrm{g}} H_{\mathrm{m}}^{\mathrm{o}}(298.15 \mathrm{~K})-T_{\mathrm{b}}$. The results obtained from this correlation agree within the experimental uncertainties with values derived with other methods.

Also, the $\Delta_{1}^{\mathrm{g}} H_{\mathrm{m}}^{\mathrm{o}}(298.15 \mathrm{~K})$ values derived with the help of chromatographic retention indices, $J_{\mathrm{x}}$, agree well with values derived for amino-alcohols with other methods. The "theoretical" $T_{\mathrm{b}}$ values and $J_{\mathrm{x}}$ values derived from both correlations, as well as the "empirical" $S F$-values, are valuable to support the level of enthalpy of vaporization derived from other methods, especially in cases where data are scarce. The experimental and "theoretical" vaporization enthalpies derived for each amino-alcohol are given in Table 2. From this table, it can be seen that for every compound agreement among $\Delta_{1}^{\mathrm{g}} H_{\mathrm{m}}^{\mathrm{o}}(298.15 \mathrm{~K})$ values, which were derived in different ways, all lie within the assigned error bars. To get more confidence and reliability, we calculated the weighted average (the uncertainty was used as a weighing factor) for of amino-alcohols given in Table 2. These values are highlighted in bold and are recommended for thermochemical calculations performed in the following section.

\section{Prediction of vaporization enthalpies of amino-alcohols with the "centerpiece" approach.}

The general idea of the "centerpiece" approach is already shown in Fig. 1. Now we apply this idea for predicting the $\Delta_{1}^{\mathrm{g}} H_{\mathrm{m}}^{\mathrm{o}}(298.15 \mathrm{~K})$ values, which are carefully evaluated in Table 2 .

First of all, let us complete the prediction shown in Fig. 1 with the help of the experimental vaporization enthalpy of 2-(methyl-amino)-ethanol $\Delta_{1}^{\mathrm{g}} H_{\mathrm{m}}^{\mathrm{o}}(298.15 \mathrm{~K})=57.7 \pm 0.2 \mathrm{~kJ}$. $\mathrm{mol}^{-1}$ given in Table 2. According to the idea, the vaporization enthalpy of 2-(phenyl-amino)-ethanol is derived by cutting the $\mathrm{CH}_{3}$ group (with its contribution to vaporization enthalpy of $6.33 \mathrm{~kJ} \cdot \mathrm{mol}^{-1}$, given in Table S4) and attaching the $\mathrm{C}_{6} \mathrm{H}_{5}$ group (with its contribution to vaporization enthalpy of $31.7 \mathrm{~kJ} \cdot \mathrm{mol}^{-1}$, given in Table S4) instead. The resulting "additive" value $\Delta_{1}^{\mathrm{g}} H_{\mathrm{m}}^{\mathrm{o}}(298.15 \mathrm{~K})=83.1 \pm 1.0 \mathrm{~kJ}$. $\mathrm{mol}^{-1}$ is very close to the transpiration result $\Delta_{1}^{\mathrm{g}} H_{\mathrm{m}}^{\mathrm{o}}(298.15$ $\mathrm{K})=82.3 \pm 0.4 \mathrm{~kJ} \cdot \mathrm{mol}^{-1}$ (see Table 2).

Another option for predicting the vaporization enthalpy of 2-(phenyl-amino)-ethanol is to start (see Fig. 3) from the experimental vaporization enthalpy of 2-(ethylamino)-ethanol $\Delta_{1}^{\mathrm{g}} H_{\mathrm{m}}^{\mathrm{o}}(298.15 \mathrm{~K})=60.8 \pm 0.2 \mathrm{~kJ} \cdot \mathrm{mol}^{-1}$ given in Table 2. In this case, in order to derive the desired value, we need to cut off the $\mathrm{CH}_{3}$ and $\mathrm{CH}_{2}$ groups and append the $\mathrm{C}_{6} \mathrm{H}_{5}$ group instead. The resulting "additive" value $\Delta_{1}^{\mathrm{g}} H_{\mathrm{m}}^{\mathrm{o}}(298.15 \mathrm{~K})=83.3 \pm 1.0 \mathrm{~kJ} \cdot \mathrm{mol}^{-1}$ is also very 


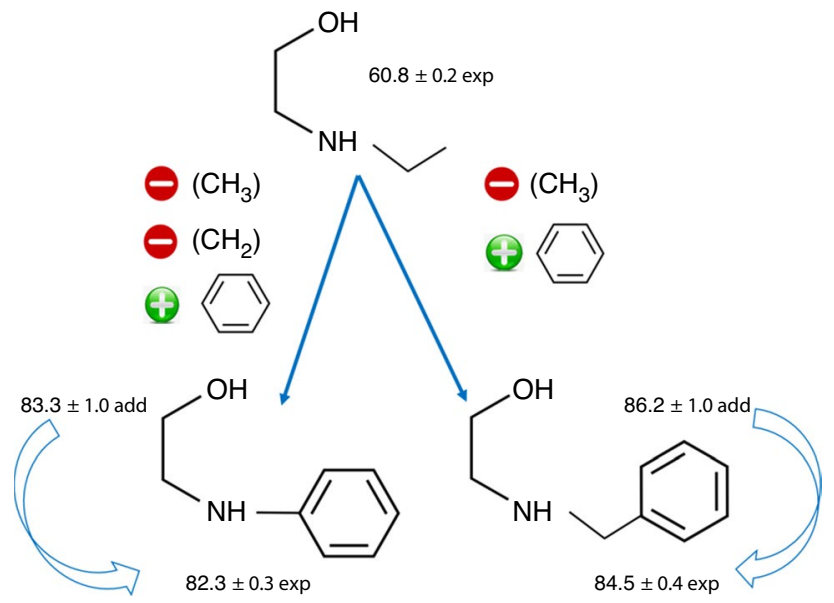

Fig. 3 Calculations of vaporization enthalpies of of 2-(phenylamino)-ethanol and of 2-(benzyl-amino)-ethanol using the "centerpiece" approach

close to the transpiration result value $\Delta_{1}^{\mathrm{g}} H_{\mathrm{m}}^{\mathrm{o}}(298.15 \mathrm{~K})=$ $82.3 \pm 0.4 \mathrm{~kJ} \cdot \mathrm{mol}^{-1}$ (see Table 2 ). It is even easier to predict the vaporization enthalpy of 2-(benzyl-amino)-ethanol starting from the same "centerpiece" 2-(ethyl-amino)ethanol as it is shown in Fig. 3. The resulting "additive" value $\Delta_{1}^{\mathrm{g}} H_{\mathrm{m}}^{\mathrm{o}}(298.15 \mathrm{~K})=86.2 \pm 1.0 \mathrm{~kJ} \cdot \mathrm{mol}^{-1}$ for 2-(benzylamino)-ethanol agrees within the combined uncertainties with the transpiration result $\Delta_{1}^{\mathrm{g}} H_{\mathrm{m}}^{\mathrm{o}}(298.15 \mathrm{~K})=84.5 \pm 0.4$ $\mathrm{kJ} \cdot \mathrm{mol}^{-1}$ (see Table 2).

The examples of the "centerpiece" approach application given in Figs. 1 and 3 show a generally possible way to obtain a reliable prediction even starting from different species. However, this method cannot be considered as universal, as the selection of the starting "centerpiece" requires some preliminary knowledge of effects that arise when substituents are placed in close proximity in the germinal or vicinal position on the alkane skeleton. This idea is demonstrated in Fig. 4. Using the "centerpiece"

Fig. 4 Calculations of vaporization enthalpy of DL-2-amino1-butanol using the "centerpiece" approach approach, the vaporization enthalpy of DL-2-amino1-butanol can be estimated starting from 2-amino-ethanol, 2-amino-1-propanol and from 2-amino-1-pentanol. Experimental vaporization enthalpies for these amino-alcohols are given in Table S5.

The four steps required to construct DL-2-amino-1-butanol from 2-amino-ethanol are shown in Fig. 4 (left). The resulting "additive" value $\Delta_{1}^{\mathrm{g}} H_{\mathrm{m}}^{\mathrm{o}}(298.15 \mathrm{~K})=67.5 \pm 1.0 \mathrm{k}$ $\mathrm{J} \cdot \mathrm{mol}^{-1}$ is clearly overestimated compared to the transpiration result $\Delta_{1}^{\mathrm{g}} H_{\mathrm{m}}^{\mathrm{o}}(298.15 \mathrm{~K})=65.5 \pm 0.5 \mathrm{~kJ} \cdot \mathrm{mol}^{-1}(\mathrm{see}$ Table 2). Maybe there are too many construction steps to get a correct result? Starting with 2-amino-1-propanol, the number of steps is considerably lower (see Fig. 4, middle). But even in this case the "additive" value $\Delta_{1}^{\mathrm{g}} H_{\mathrm{m}}^{\mathrm{o}}(298.15 \mathrm{~K})$ $=62.6 \pm 1.0 \mathrm{~kJ} \cdot \mathrm{mol}^{-1}$ is clearly underestimated compared to the transpiration result $\Delta_{1}^{\mathrm{g}} H_{\mathrm{m}}^{\mathrm{o}}(298.15 \mathrm{~K})=65.5 \pm 0.5 \mathrm{~kJ}$ $\cdot \mathrm{mol}^{-1}$ (see Table 2). What is wrong with the centerpiece approach? Starting with 2-amino-1-pentanol, we also need only one step to construct DL-2-amino-1-butanol (see Fig. 4, right). We can finally get the "additive" result $\Delta_{1}^{\mathrm{g}} H_{\mathrm{m}}^{\mathrm{o}}(298.15 \mathrm{~K})=64.6 \pm 1.0 \mathrm{~kJ} \cdot \mathrm{mol}^{-1}$ comparable to the experiment. What can be the reason for the trial and error observed in all three cases?

At first glance, none of the three starting molecules, namely 2-amino-ethanol, 2-amino-1-propanol, and 2-amino-1-pentanol, are very different. But it is not correct. It is known that the energetics of aminoalcohols and 1,2-alkanediols are determined by the intra-molecular hydrogen bonding $[24,25]$. Let us first consider 1,2-alkanediols, for which a plausible explanation was given in our previous work [25]. With the 1,2-alkanediols, an unusual sequence of the $\Delta_{1}^{\mathrm{g}} H_{\mathrm{m}}^{\mathrm{o}}(298.15 \mathrm{~K})$-values was observed with an increasing chain length (in $\mathrm{kJ} \cdot \mathrm{mol}^{-1}$ ): 1,2-ethanediol $(66.0 \pm 0.2), 1,2$-propanediol $(64.5 \pm 0.2), 1,2$-butanediol (70.4 \pm 0.3$), 1,2$-pentanediol (74.6 \pm 0.3$)$, and 1,2-hexanediol (78.7 \pm 0.3$)$ [25]. Such behavior of 1,2-alkanediols can be explained by a strong intra-HB, which obviously dominates in the liquid state. In the case of 1,2-propanediol,

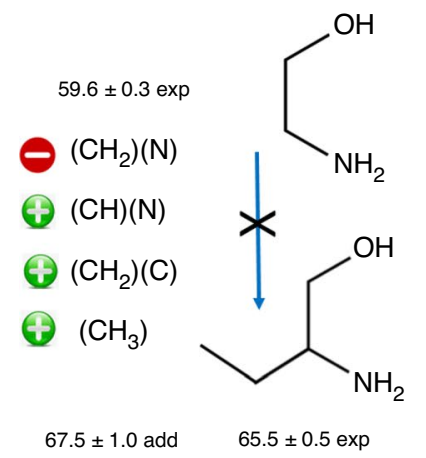

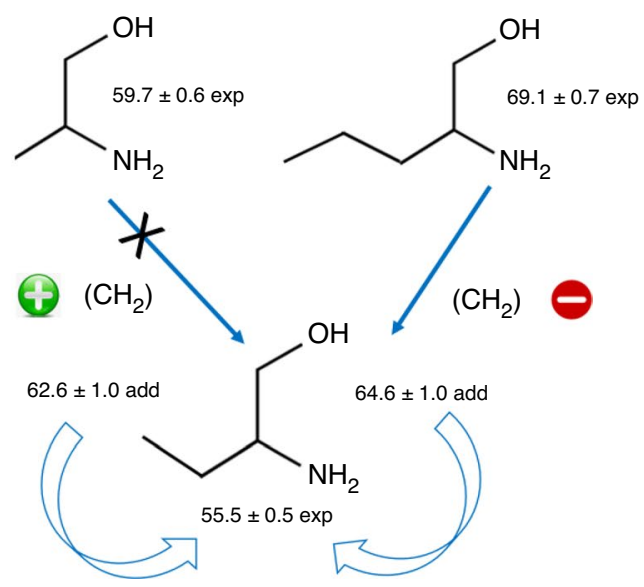




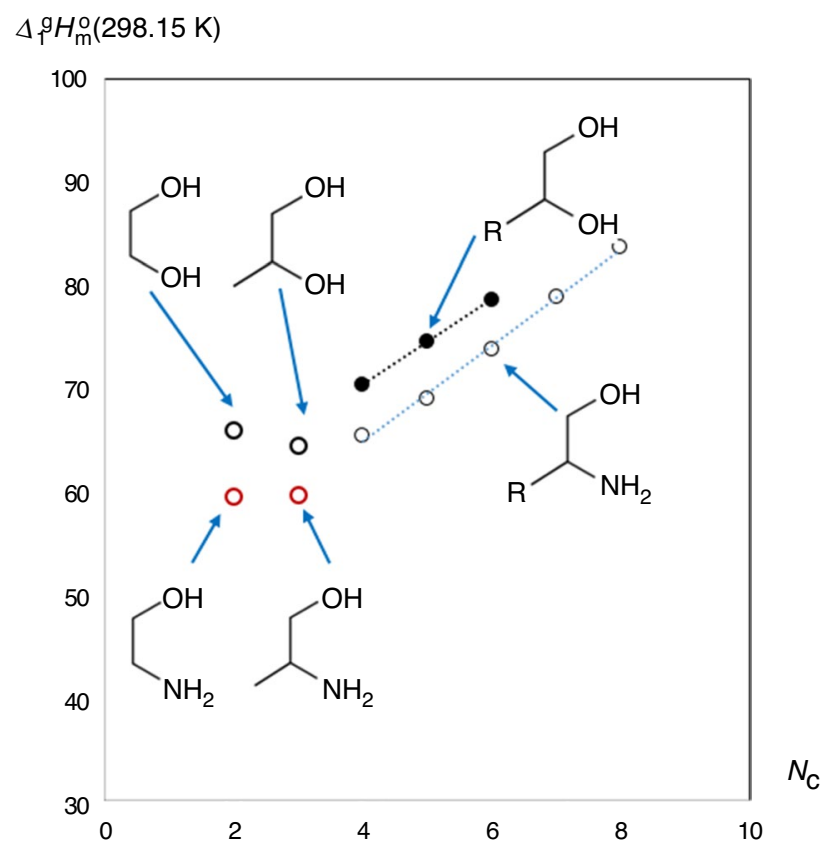

Fig. 5 Chain-length dependence of vaporization enthalpies $\Delta_{1}^{\mathrm{g}} H_{\mathrm{m}}^{\mathrm{o}}$ $(298.15 \mathrm{~K}) / \mathrm{kJ} \cdot \mathrm{mol}^{-1}$ in 1,2-alkanediols and in 2-amino-1-alkanols. $N_{\mathrm{C}}$-is the total number of C-atoms in the molecule

the addition of an external methyl group leads to a steric hindrance to the formation of an intra-HB. Because of this steric hindrance, the enthalpy of vaporization of 1,2-propanediol is reduced compared to 1,2-ethanediol. However, an attachment of the next alkyl fragments to the 1,2-ethanediol unit does not have the same effect as with the first substitution, since the added fragments are further away from the hydroxyl groups, which form the intra-HB. With increasing chain length in 1,2-butanediol and 1,2-pentanediol, the substitution effect on the intra-HB is already compensated and the $\Delta_{1}^{\mathrm{g}} H_{\mathrm{m}}^{\mathrm{o}}(298.15 \mathrm{~K})$ values become linearly dependent on the chain length (see Fig. 5).

As can be seen in Fig. 5, the trend similar to that of 1,2-alkanediols applies to aminoalcohols: the strongest intra-HB is in 2-amino-ethanol. However, already in 2-amino-1-propanol, the $\mathrm{CH}_{3}$ group, which is located in the close proximity of the amino-group, reduces the strength of the intra-HB for steric reasons. Similar to 1,2-alkanediols, an attachment of the next alkyl fragments to 2-amino-ethanol does not have the same effect as with the first substitution. As a consequence, the vaporization enthalpies of 2-amino-ethanol and 2-amino-1-propanol are out of the linear correlation shown in Fig. 5. The linearity of the chain-length dependence of the enthalpy of vaporization begins with 2-amino-1-butanol. Such a peculiar energetic behavior that has been observed in aminoalcohols can now explain the trial and error observed with three "centerpieces" shown in Fig. 4. Indeed, using

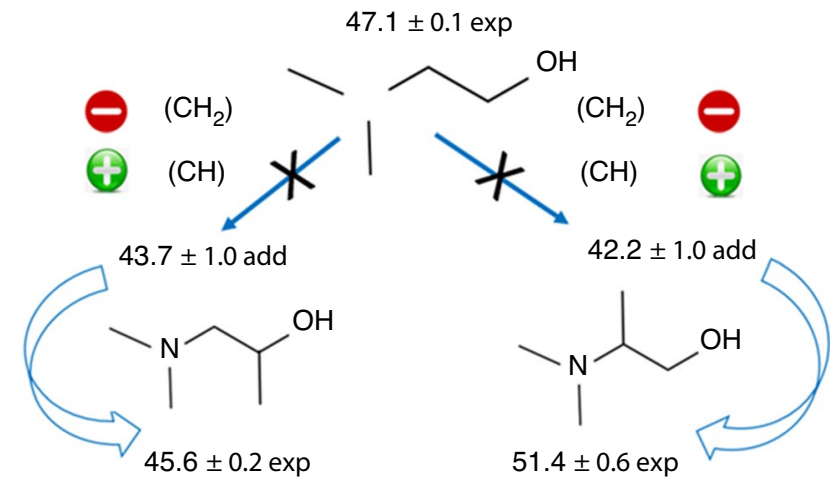

Fig. 6 Calculations of vaporization enthalpies of 2-(dimethyl-amino)1-propanol and 1-(dimethyl-amino)-2-propanol using the "centerpiece" approach

2-amino-ethanol and 2-amino-1-propanol to predict the enthalpy of vaporization of 2-amino-1-butanol could be considered as a typical "error," as both molecules have much more individual characteristics than is applicable for group additivity. In contrast, using the 2-amino-1-pentanol as the "centerpieces" is optimal for the prediction, since according to Fig. 5, this molecule is already out of perturbation specific for the smaller homologues.

Another important factor for the selection of the "centerpiece" molecule is illustrated in Fig. 6. Let us try to predict vaporization enthalpies of two branched aminoalcohols: enthalpies of 2-(dimethyl-amino)-1-propanol and 1-(dimethyl-amino)-2-propanol starting from $\Delta_{1}^{\mathrm{g}} H_{\mathrm{m}}^{\mathrm{o}}(298$. $15 \mathrm{~K})=47.1 \pm 0.1 \mathrm{~kJ} \cdot \mathrm{mol}^{-1}$ [26] of 2-(dimethyl-amino)ethanol as the "centerpiece" molecule. In both cases, we just need to replace the $\mathrm{CH}_{2}$ group with the $\mathrm{CH}$ group and add the contribution for the $\mathrm{CH}_{3}$ group as it is shown in Fig. 6. In both cases, however, the "additive" results do not agree with the experimental values evaluated in Table 2.

The obvious reason for the observed disagreement is the appearance of steric repulsions between substituents, since they occur in close proximity on the small skeleton. Due to these repulsions, the tightness of the packaging of the molecules in the liquid is unique for each type of branching. Therefore, the correct selection of the "centerpiece" molecule is hardly possible.

The consequence for proper use of the "centerpiece" approach is that the small branched molecules and molecules with strong steric interactions (e.g., 2-amino2-methyl-1-propanol) must be excluded from application. Nonetheless, we are systematically testing the "centerpiece" approach with different types of organic and metal-organic compounds. The reliable results have been already obtained for aldehydes and esters [27], for substituted benzenes [6, 7], and for tris(beta-diketonato)iron complexes [28]

In summary, the compilation of experimental results evaluated in Table 2 made it possible to validate three 
different approaches (SF, $T_{\mathrm{b}}$, and "centerpiece") in order to reliably assess the $\Delta_{1}^{\mathrm{g}} H_{\mathrm{m}}^{\mathrm{o}}(298.15 \mathrm{~K})$ values for organic and metal-organic compounds.

Supplementary Information The online version contains supplementary material available at https://doi.org/10.1007/s10973-021-11115-4.

Acknowledgements Authors gratefully acknowledge financial support from the Government of Russian Federation (Decree No. 220 of 9 April 2010), Agreement No. 14.Z50.31.0038. SPV and IVA Acknowledge financial support from German Science Foundation (Grant VE 265/12-1 to SPV).

Funding Open Access funding enabled and organized by Projekt DEAL.

Open Access This article is licensed under a Creative Commons Attribution 4.0 International License, which permits use, sharing, adaptation, distribution and reproduction in any medium or format, as long as you give appropriate credit to the original author(s) and the source, provide a link to the Creative Commons licence, and indicate if changes were made. The images or other third party material in this article are included in the article's Creative Commons licence, unless indicated otherwise in a credit line to the material. If material is not included in the article's Creative Commons licence and your intended use is not permitted by statutory regulation or exceeds the permitted use, you will need to obtain permission directly from the copyright holder. To view a copy of this licence, visit http://creativecommons.org/licenses/by/4.0/.

\section{References}

1. Benson SW. Thermochemical kinetics methods for the estimation of thermochemical data and rate parameters. New York: Wiley; 1968.

2. Ducros M, Gruson JF, Sannier H. Estimation des enthalpies de vaporisation des composes organiques liquides Partie 1 Applications aux alcanes cycloalcanes, alcenes, hydrocarbures benzeniques, alcools, alcanes thiols, chloro et bromoalcanes, nitriles, esters, acides et aldehydes. Thermochim Acta. 1980;36:39-65.

3. Benson SW. New methods for estimating the heats of formation, heat capacities, and entropies of liquids and gases. J Phys Chem A. 1999;103:11481-5.

4. Verevkin SP, Emel'yanenko VN, Diky V, Muzny CD, Chirico RD, Frenkel M. New group-contribution approach to thermochemical properties of organic compounds: hydrocarbons and oxygencontaining compounds. J Phys Chem Ref Data. 2013;42:033102.

5. Verevkin SP, Emel'yanenko VN, Nagrimanov RN. Nearest and non nearest neighbor interactions between substituents in the benzene ring experimental and theoretical study of functionally substituted benzamides. J Phys Chem A. 2016;120:9867-77.

6. Verevkin SP, Konnova ME, Emel'yanenko VN, Pimerzin AA. Weaving a network of reliable thermochemistry around lignin building blocks: vanillin and its isomers. J Chem Thermodyn. 2021;157:106362.

7. Verevkin SP, Turovtsev VV, Andreeva IV, Orlov YD, Pimerzin AA. Webbing a network of reliable thermochemistry around lignin building blocks: tri-methoxy-benzenes. RSC Adv. 2021;11:10727-37.

8. Steele WV, Chirico RD, Knipmeyer SE, Nguyen A. Vapor pressure, heat capacity, and density along the saturation line, measurements for cyclohexanol, 2-cyclohexen-1-one, 1,2-dichloropropane, 1,4-di-tert-butylbenzene, 2-ethylhexanoic acid, 2-(methylamino) ethanol, perfluoro-n-heptane, and sulfolane. J Chem Eng Data. 1997;42:1021-36.
9. Kapteina S, Slowik K, Verevkin SP, Heintz A. Vapor pressures and vaporization enthalpies of a series of ethanolamines. J Chem Eng Data. 2005;50:398-402.

10. Kulikov D, Verevkin SP, Heintz A. Enthalpies of vaporization of a series of linear aliphatic alcohols. experimental measurements and application of the ERAS-model for their prediction. Fluid Phase Equilib. 2001;192:187-207.

11. Verevkin SP, Emel'yanenko VN. Transpiration method: vapour pressures and enthalpies of vaporization of some low-boiling esters. Fluid Phase Equilib. 2008;266:64-75.

12. Verevkin SP, Sazonova AY, Emel yanenko VN, Zaitsau DH, Varfolomeev MA, Solomonov BN, Zherikova KV. Thermochemistry of halogen-substituted methylbenzenes. J Chem Eng Data. 2015;60:89-103.

13. Emel'yanenko VN, Verevkin SP. Benchmark thermodynamic properties of 1,3-propanediol: comprehensive experimental and theoretical study. J Chem Thermodyn. 2015;85:111-9.

14. Stull DR. Vapor Pressure of Pure Substances. Organic and Inorganic Compounds. Ind Eng Chem. 1947;39:517-40.

15. Razzouk A, Hajjaji A, Mokbel I, Mougin P, Jose J. Experimental vapor pressures of 1,2-bis(dimethylamino)ethane, 1-methylmorpholine, 1,2-bis(2-aminoethoxy)ethane and N-benzylethanolamine between 273.18 and 364.97K. Fluid Phase Equilib. 2009;282:11-3.

16. SciFinder® https://scifinder.cas.org/. Accessed 10 Sep 2021.

17. NIST Chemistry WebBook. https://webbook.nist.gov/chemistry/. Accessed 10 Sep 2021

18. PubChem. https://pubchem.ncbi.nlm.nih.gov/. Accessed 10 Sep 2021.

19. GuideChem. https://www.guidechem.com/. Accessed 10 Sep 2021.

20. Verevkin SP, Andreeva IV, Pimerzin AA. Evaluation of vaporization thermodynamics of pure amino-alcohols. J Mol Liquids. 2021;335:1165682021. https://doi.org/10.1016/j.molliq.2021.116568.

21. Kovats E. Gas-chromatographische Charakterisierung Organischer Verbindungen. Teil 1: Retentionsindices Aliphatischer Halogenide, Alkohole, Aldehyde und Ketone. Helv Chim Acta. 1958;41:1915-32

22. Verevkin SP. Vapour pressures and enthalpies of vaporization of a series of the linear n-alkyl-benzenes. J Chem Thermodyn. 2006;38:1111-23.

23. Liu F, Liang Y, Cao C, Zhou N. Theoretical prediction of the Kovat's retention index for oxygen-containing organic compounds using novel topological indices. Anal Chim Acta. 2007;594:279-89.

24. Kelterer A-M, Ramek M. Intramolecular hydrogen bonding in 2-aminoethanol, 3-aminopropanol and 4-aminobutanol. J Mol Struct (Thoechem). 1991;232:189-201.

25. Verevkin SP. Determination of vapor pressures and enthalpies of vaporization of 1,2-alkanediols. Fluid Phase Equilib. 2004;224:23-9.

26. Chiali-Baba-Ahmed N, Dergal F, Negadi L, Mokbel I. Measurement and correlation of the (vapor + liquid) equilibria of pure 4-ethylmorpholine, 1,2-dimethylisopropylamine and N, N-dimethylethanolamine, and their binary aqueous solutions. J Chem Thermodyn. 2013;63:44-51.

27. Siewert R, Verevkin SP, Zaitsau DH, Emel'yanenko VN. Biomass valorization: thermodynamics of the Guerbet condensation reaction. J Chem Eng Data. 2019;64:4904-14.

28. Zherikova KV, Verevkin SP. Error or exemption to the rule? development of a diagnostic check for thermochemistry of metalorganic compounds. RSC Adv. 2020;10:38158-73.

Publisher's Note Springer Nature remains neutral with regard to jurisdictional claims in published maps and institutional affiliations. 